\title{
A Note on Punctuation
}

ouble quotation marks indicate citations of other authors, terms used
in a commonly accepted sense to which I am referring as quotations
(though no particular source may be provided), or, in some few cases,
phrases cited from earlier parts of the book. Single marks, except where
they indicate a quotation within a quotation, are used for other empha-
ses-most often either to indicate the inappropriateness of some habitu-
ally used term or to signal that a term taken from one discursive logic (or
class of discourse) is being unavoidably but unsuitably applied to a differ-
ent such logic. 

The Uncertainty of Analysis 
